\title{
3D Facial Action Units Recognition for Emotional Expression
}

\author{
Norhaida Hussain, Hamimah Ujir, Irwandi Hipiny, Jacey-Lynn Minoi
}

\begin{abstract}
The muscular activities caused the activation of facial action units (AUs) when a facial expression is shown by a human face. This paper presents the methods to recognize $A U$ using a distance feature between facial points which activates the muscles. The seven $A U$ involved are $A U 1, A U 4, A U 6, A U 12$, AU15, AU17 and AU25 that characterizes a happy and sad expression. The recognition is performed on each $A U$ according to the rules defined based on the distance of each facial point. The facial distances chosen are computed from twelve salient facial points. Then the facial distances are trained using Support Vector Machine (SVM) and Neural Network (NN). Classification result using SVM is presented with several different SVM kernels while result using $N N$ is presented for each training, validation and testing phase. By using any SVM kernels, it is consistent that AUs that are corresponded to sad expression has a high recognition compared to happy expression. The highest average kernel performance across AUs is $93 \%$, scored by quadratic kernel. Best results for NN across AUs is for AU25 (Lips parted) with lowest CE (0.38\%) and 0\% incorrect classification.
\end{abstract}

Index Terms: $3 D A U$ recognition, facial action unit's recognition, facial expression, Support Vector Machine, Neural Network

\section{INTRODUCTION}

Facial expressions are significant to non-verbal communications as they provide varies information such as emotional state, attitudes, personality and age. The analysis of facial expression plays a major role in emotional recognition that contributes toward the development of the Human-Computer Interaction (HCI) system, which could be applied in the area such as neurology, lie detection, intelligent environments and paralinguistic communication [1]. As machines become more involve in human daily life, they need to become more intelligent to understand human moods and emotions [29]. facial muscles that caused temporary deformation of the facial surface, including the geometry and texture characteristics [2]. Action Unit (AU) defined in the Facial Action Coding System (FACS) [6] is a component of facial expression triggered by distinctive facial muscle that lies below the facial skin.

Revised Version Manuscript Received on August 19, 2019.

Norhaida Hussain, Department of Information Technology and Communication, Politeknik Kuching, Malaysia,(email: norhaida@poliku.edu.my)

Hamimah Ujir, Faculty of Computer Science and Information Technology, Universiti Malaysia Sarawak, Malaysia, (email:uhamimah@unimas.my)

Irwandi Hipiny, Faculty of Computer Science and Information Technology, Universiti Malaysia Sarawak, Malaysia, (email: mhihipni@unimas.my)

Jacey-Lynn Minoi, Faculty of Computer Science and Information Technology, Universiti Malaysia Sarawak, Malaysia,(email: jacey@unimas.my)
Facial expression is initiated by the contractions of distinct

There are six basic emotional facial expressions acknowledged by psychologists; happy, surprise, angry, fear, sad and disgust. However, the six emotions partially represent only several human facial expressions. Due to this, $\mathrm{AU}$ recognition is preferable so that the activated muscles are recognized rather than the expression itself.

Apart from recognizing facial expressions, another interesting study in face processing area is facial expression intensity estimation. Human tend to show simple facial motion such as tightening the lips in anger or obliquely lowering the lip corners in sadness [18]. This is because of human facial expressions can vary much in intensity e.g.: from uneasiness and mild fear to angst and panic [31]. Lower intensity of certain facial expression might not be familiar to some people. Besides, intensity level of a facial expression is important as it will lead to a false impression of people's emotion if misinterpreted. For example, the smiling face with low intensity can be easily misinterpreted as a neutral facial expression [19]. [20] listed several reasons on why we need to estimate facial expression intensity. One of the reasons is there are differences in terms of the way that different individuals express the same facial expression, such as transition speed, magnitude and duration. However, the task of facial expression intensity estimation remains less focused compared to facial expression classification itself. Through AU recognition, facial expression intensity could be measured accordingly as AU is related to the facial muscle. Fig. 1 shows six basic facial expressions with different four intensity levels. As we go up the intensity level, the facial expression is more intense and the facial points' displacement is apparent.

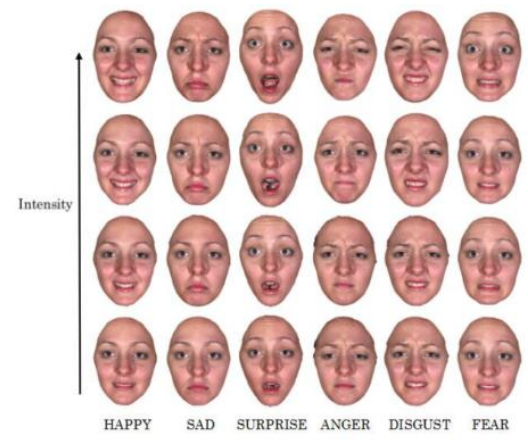

Fig. 1.Intensity Level of Facial Expression in BU-3DFE Database [28] 
This paper presents the study of the AU presence in corresponds to happy and sad expression using two different classification approaches. The first objective is to compute $3 \mathrm{D}$ facial distances for happy and sad expression. The second objective is to classify the AU using Support Vector Machines (SVM) and Neural Networks (NN). Section 2 describes the related works in this field, followed by a discussion on the AU recognition process in section 3. Then, the geometric facial features are discussed in section 4 . Section 5 is about the experiments.

\section{RELATED WORKS}

Table 1.Four temporal phases in facial action [10]

\begin{tabular}{|l|l|}
\hline $\begin{array}{c}\text { Temporal } \\
\text { Phase }\end{array}$ & \multicolumn{1}{c|}{ Description } \\
\hline Onset & $\begin{array}{l}\text { Facial muscles are contracting, facial } \\
\text { appearance changes gradually when } \\
\text { facial action increases. }\end{array}$ \\
\hline Apex & $\begin{array}{l}\text { The peak of facial action and facial } \\
\text { appearance stop changing. }\end{array}$ \\
\hline Offset & $\begin{array}{l}\text { The muscles are contracting to relax, and } \\
\text { the face returns to its neutral appearance } \\
\text { at slow pace. }\end{array}$ \\
\hline Neutral & $\begin{array}{l}\text { There is no sign of facial action } \\
\text { activation. }\end{array}$ \\
\hline
\end{tabular}

The presence of AUs are only at certain duration throughout the full facial expression, which is from neutral to offset phase. The four temporal phases are described in Table 1. To enable the analysis of facial action temporal phases, there are four temporal segments need to be detected; 1 neutral to onset, 2- onset to apex, 3-apex to offset, 4- offset to neutral. When the transition from each temporal segment is captured, the intensity of AU could be computed at each of the temporal segment for further improvement in facial expression recognition.

To improve the recognition performance of each $\mathrm{AU}$, selecting salient facial feature points is very important since they represent the geometric property of AU activation. 3D facial distances are used in [15] to measure facial expression intensity. From these phases, the monitoring of salient facial features is carried out and the intensity of shown facial expression could be detected. In [12] [14], surface normal is computed using the provided 3D facial points to classify facial expression.

On the other hand, the classifier algorithms used to classify the AUs also plays a significant role in getting good results. Thus, it is important to decide which classifier that suitable to recognize the AUs. There are many techniques used to classify AUs using 3D facial data such as SVM [3], Hidden Markov Model (HMM) [4] and Local Binary Pattern (LBP) [5].

All basic facial expressions involve different facial muscles. The AUs correspond to all basic facial expressions presented in Table 2 are based on previous studies by [6][7][8]. These AUs are activated by predefined facial features and normally the distances between two or more facial points are the facial features used. These distances are the extracted features used to recognize the activation of AU during any facial expression. Based on Table 2, happy and sad expression involved a different set of AUs. For example, according to [6], only AU6 and AU12 present during happy expression. On the hand, in [7] and [8], AU25 is said to be present as well when happy expression occurred. Table 3 defines AU which involved in a Happy and Sad expression.

Table 2.Emotions and Corresponding AUs.

\begin{tabular}{|c|c|c|c|}
\hline $\begin{array}{c}\text { Action } \\
\text { Units }\end{array}$ & $\begin{array}{c}\text { Ekman \& } \\
\text { Friesan[6] }\end{array}$ & $\begin{array}{c}\text { Lucey et } \\
\text { al [7] }\end{array}$ & $\begin{array}{c}\text { Karthick } \\
\text { et al [8] }\end{array}$ \\
\hline Happy & $6+12$ & $6+12+25$ & $6+12+25$ \\
\hline Sad & $1+4+15$ & $1+2+4+15+$ & $1+4+15+1$ \\
& & 17 & 7 \\
\hline Surprise & $1+2+5 B+26$ & $1+2+5+25+$ & $5+26+27+$ \\
& & 27 & $1+2$ \\
\hline Disgust & $9+15+16$ & $1+4+15+17$ & $9+17$ \\
\hline Anger & $4+5+7+23$ & $4+5+15+17$ & $4+5+7+23$ \\
& & & +24 \\
\hline Fear & $1+2+4+5+20$ & $1+4+7+20$ & $4+1+5+7$ \\
& +26 & & \\
\hline
\end{tabular}

Table 3.AUs Definition [9].

\begin{tabular}{|l|l|}
\hline AU & Definition \\
\hline AU1 & Raised inner eyebrow \\
\hline AU4 & Eyebrows drawn together, lowered eyebrows \\
\hline AU6 & Raised cheek, compressed eyelid \\
\hline AU12 & Mouth corners pulled up \\
\hline AU15 & Mouth corners downward \\
\hline AU17 & Chin raised \\
\hline AU25 & Lips parted \\
\hline
\end{tabular}

The AU recognition rule could affect the accuracy rate of the AU presence during an occurrence of any facial expression. In the previous work by [9], 30 FACS rules and 30 expert system rules are mapped to recognize the activation of a single AU. Each AU is encoded with certain rule based on the distance of facial points, measured between expression and expressionless face. The encoded rules which related to the AUs, correspond to happy and sad facial expression, are described in [9]. The rules are revised to ensure it is suitable to be employed with the selected facial points used in this project.

A total of 27 AUs was successfully detected with an average recognition rate of $86.6 \%$ using 24 rules developed for a profile-view face of face image sequences in [10]. Also, 95\% average accuracy in detecting temporal segment of facial expression [10]. AU recognition rules were developed based on 15 fiducial points on a profile-view face image. In addition, the rule to detect the temporal segments of AUs is also included in the AU recognition rule. There are 7 rules presented in Table 4. Based on Table 4, Rule 1 is defined to detect the presence of AU1 and this AU is only considered as present when a position of a facial feature located at the inner corner of an eyebrow, is moving up or down more than the threshold value which is set to be equal to 1 .

Published By: 
Table 4.Rules to Recognise the Presence of AU1, AU4, AU6, AU12, AU15, AU17 and AU25 [10]

\begin{tabular}{|c|c|}
\hline Rule & Description \\
\hline Rule 1 & IFup/down $(\mathrm{P} 12)>\varepsilon$ THEN AU1 - p \\
\hline Rule 3 & $\begin{array}{l}\text { IF }[\text { inc/dec }(\mathrm{P} 2 \mathrm{P} 12)]>\varepsilon A N D i n c / \operatorname{dec}(\mathrm{P} 2 \mathrm{P} 12) \leq \mathrm{T} 1 \\
\text { THEN AU4 - p }\end{array}$ \\
\hline Rule 5 & $\begin{array}{l}\text { IF }[\text { inc/dec }(\mathrm{P} 13 \mathrm{P} 14)]_{\mathrm{P}}>\varepsilon \text { ANDinc/dec }(\mathrm{P} 13 \mathrm{P} 14) \leq \\
\mathrm{T} 1 \text { ANDup/down }(\mathrm{P} 7)>\varepsilon \\
\text { THEN AU6 - } \mathrm{p}\end{array}$ \\
\hline Rule 9 & $\begin{array}{l}\text { IFup/down }(\mathrm{P} 7)>\varepsilon \text { ANDinc/dec(P5P7) }]_{<\varepsilon} T H E N \\
\text { AU12-p }\end{array}$ \\
\hline Rule 11 & IF up/down $(\mathrm{P} 7)<\varepsilon$ THEN AU15-p \\
\hline Rule 13 & $\begin{array}{l}\text { IF NOT (AU28 OR AU 28t OR AU28b) AND } \\
\text { inc/dec(P10P15)> }>\text { THEN AU17- }\end{array}$ \\
\hline Rule 18 & $\begin{array}{l}I F[\text { inc/dec }(\mathrm{P} 6 \mathrm{P} 8)]_{\mathrm{t}}<\varepsilon \text { ANDinc/dec }(\mathrm{P} 4 \mathrm{P} 10) \geq \varepsilon \\
\text { THEN AU25 }-\mathrm{p}\end{array}$ \\
\hline
\end{tabular}

Many classification algorithms that can be used in facial expression or AU recognition such as boosting, Support Vector Machines, Neural Network, nearest neighbour, Naive Bayes and random forests.

There are numerous studies using SVM for facial expression recognition. This is due to the ability to select appropriate kernel function that allowed further adjustment and optimization of SVM [23]. They obtained accuracy of $87.9 \%$ for facial expression recognition after kernel optimization, average accuracy of $69.29 \%$ for facial actions recognition in [24]. [25] has used BU-3DFE [21] database to recognize six facial expressions and has achieved a recognition rate at average of $81.9 \%$ and $79.9 \%$ by SVM with linear kernel and Radial Basis Function (RBF) kernel respectively.

[22] trained the neural network separately for AUs in upper face and lower face region using only one hidden layer. The recognition rate achieved is $89.4 \%$ for AU1, AU2, AU4, AU5, AU6 and AU7. The recognition rate achieved is $89.4 \%$ for AU1, AU2, AU4, AU5, AU6 and AU7. The accuracy of facial expression recognition achieved in [26] is $80 \%$ for happy expression, $75 \%$ for sad expression, $80 \%$ for surprise expression, $70 \%$ for angry expression which gives average of $76 \%$ using neural network. While, [27] achieved recognition rate of $100 \%$ for all expression except for sad which only achieved $88.89 \%$.

\section{AU RECOGNITION PROCESS}

By concentrating on a region of interest (ROI) help to recognize an AU. For example, to measure human task performance, ROI of gaze is used [16] [17]. Our ROI in this study is the region of the facial points that involve in face muscle deformation. There are seven main processes involved in this project, as described in Fig. 2. The next process after facial point's extraction is the distance measurement between two facial points.

The 3D face data enables the study on wider range of facial expression due to the fact that facial surface can be measured directly as contradict to 2D data [30]. In this work, we used a $3 \mathrm{D}$ facial expression database developed by researchers from Binghamton University, known as BU-3DFE [21]. It consists of seven basic facial expression (i.e. neutral, happy, sad, disgust, anger, surprise and fear) captured on 60 different people using $3 \mathrm{D}$ face scanner. The database is created to encourage the research in $3 \mathrm{D}$ face recognition with the main intention to model spontaneous facial expression. The facial data were captured from 100 subjects that make the 2500 facial data. This database is diverse is term of ethnicity and gender. It contains 56 female subjects and 44 male subjects' age from 18 years to 70 years old.

The 3D feature points are extracted from BU-3DFE [21] database to obtain twelve facial points that correspond to a happy and sad expression. For each AU, the properties are specified based on increasing decreasing of distance value between selected facial points.

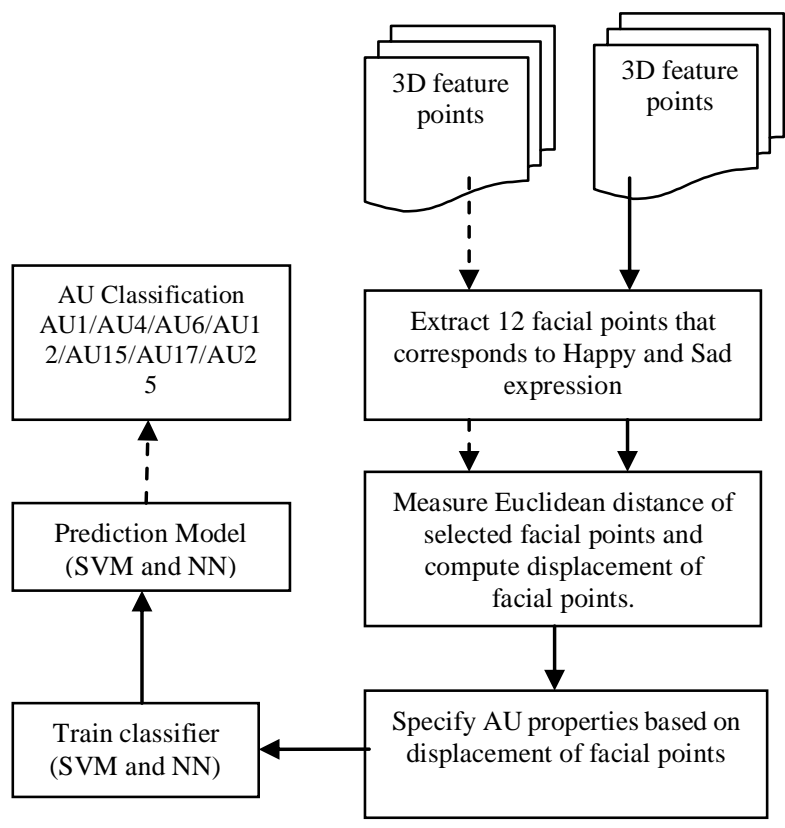

Fig. 2. The block diagram of process

The next process is to train the learning classifier to obtain the prediction model. There are many classification algorithms that are used in the facial expression and AU recognition and it is mentioned in the previous section. In this project, we have decided to use two classification techniques, which is SVM and NN.

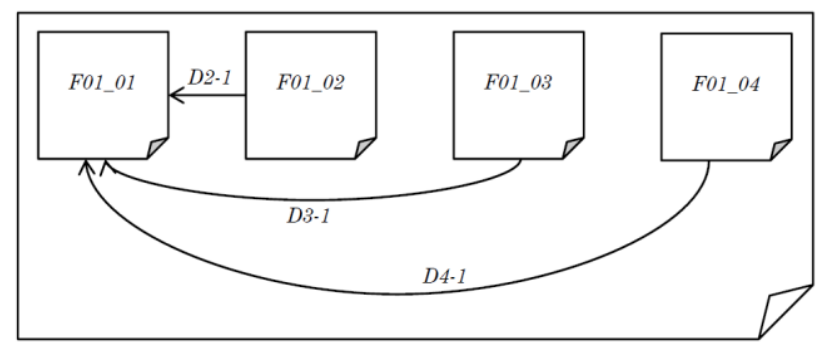

Fig. 3. Illustration of displacement facial points' measurement

The input to the learning classifier is the distance value for each facial point. To obtain the displacement value for each facial point, the distance is computed, and the value is compared to the first intensity 
level, F01_01. Based on figure 3, facial data labelled as F01_01, F01_02, F01_03 and F01_04 are the intensity level in the first facial data. The distances for all twelve properties are computed before the displacement of facial points between frames F01_02 to F01_01, F01_03 to F01_01 and F01_04 to F01_01.

As shown in figure 3 , the displacement of facial points in the second intensity level (F01_02) is computed, and the distances are saved as D2-1. Then the displacement of facial points in the third intensity level (F01_03) is computed, and the results are saved as D3-1. Lastly, the displacement of facial points in the fourth intensity level (F01_04) is computed, and the distances are saved as D4-1. The distances are measured for all facial data which consists of 30 facial data for happy and 30 facial data for sad expression.

The next process is to identify the properties for each AU according to the displacement of facial points measured earlier. The distance and corresponding AU label are the input to the learning classifier during training. The $\mathrm{AU}$ is labelled either as 1 or 0 that describes a presence and not a presence of AU, respectively. For AU1, AU6 and AU25, the input to the learning classifier is the displacement distance, and the expected output is the AU labelled as 1 or 0 . Meanwhile, for AU4, AU12 and AU17, there are two distance values with one anticipated output. The expected output which is the AU label is obtained by using logical AND operator for both properties. If property 1 and property 2 are equal to $1, \mathrm{AU}$ is labelled as 1 , which indicates $\mathrm{AU}$ is the presence. If either property equal to 1 , AU label is set to 0 . Both properties must be satisfied for the AU to be presented. Lastly, AU15 has three distance properties as input to the classifiers.

$\mathrm{NN}$ is trained using the back-propagation algorithm, and the training process stops when generalization stops improving. However, if the per cent error is high, the network is trained again to achieve better results with fewer missed classifications. Three different kernels are used in SVM classification.

\section{GEOMETRIC FACIAL FEATURES}

The facial expression analysis in $3 \mathrm{D}$ facial could be performed by extracting different features such as geometric, which includes facial points and position of the facial points. Happy and sad facial expressions involve just several number of AUs, which means happy and sad expressions only involve certain facial points. There are twelve facial points extracted from the original 83 facial points provided in BU-3DFE [21] database. These points are corresponding to the seven AUs to be detected which covers both upper and lower face AUs. Upper face AUs are AU1, AU4 and AU6 while lower face AUs are AU12, AU15, AU17 and AU25. Geometric features are computed between the selected facial points. Figure 4 shows the distance properties for happy expression and distance properties for sad expression.

The idea of selecting facial points only one side of the face is based on [11]. The selection of facial points on the other side is considered as repetitive selection due to the symmetrical property of human face. The position of selected facial points is observed for each intensity level of BU-3DFE facial data. In this study, one extra point which is point 76 that represents a tip of the chin is added. Tip of the chin is used to determine the property of AU17 (chin raised) which occurs during sad expression. The position of chosen facial points is observed for each frame.

The Euclidean distance is computed between the selected facial points for each frame in happy and sad facial expression. The similar distance calculation is found in [12][13][15]. The formula to compute the Euclidean distance, $\mathrm{ED}$, of point label $\mathrm{x}$ and $\mathrm{y}$ is:

$$
E D(x, y)=\sqrt{\left(x_{1}-y_{1}\right)^{2}+\left(x_{2}-y_{2}\right)^{2}+\left(x_{3}-y_{3}\right)^{2}}
$$

The difference of distance for each distance property is observed from the second frame until the last frame. The difference of distance between those frames is compared to the first frame. The increased $(d+)$ and decreased (d-) of those distances is observed to recognize AU as shown in Table 5.

Table 5. Emotions and Corresponding AUs

\begin{tabular}{|c|c|c|}
\hline Facial Expression & $\mathbf{A U}$ & Distance Properties \\
\hline \multirow[t]{4}{*}{ Нарру } & AU6 & - P11P15 (d-) \\
\hline & AU12 & - P55P82 (d-) \\
\hline & & - P45P55 (d-) \\
\hline & AU25 & - P52P58 (d+) \\
\hline \multirow[t]{8}{*}{ Sad } & AU1 & - P9P27 (d+) \\
\hline & $\overline{\text { AU4 }}$ & - P9P27 (d-) \\
\hline & & - P27P28 (d-) \\
\hline & AU15 & - P45P55 (d+) \\
\hline & & - P55P82 (d+) \\
\hline & & - P9P55 (d+) \\
\hline & AU17 & - P76P82 (d+) \\
\hline & & - P76P45 (d+) \\
\hline
\end{tabular}

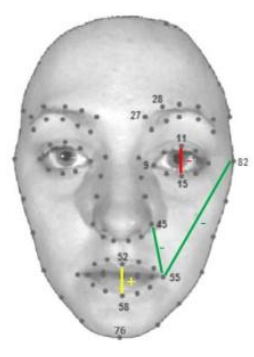

(a)

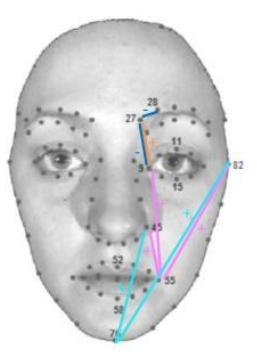

(b)
Fig. 4. The distance properties (a) Distance properties for Happy expression (b) Distance properties for Sad expression.

The presence of $\mathrm{AU}$ is set based on distance properties shown in Table 3. For example, AU12 (lip corner pulled up) is activated when both distance properties are TRUE, and the value is set to 1 . If both properties are not achieved, the value is set to 0 , which means $\mathrm{AU}$ is not activated. AU12 is activated when there is a decreased distance of P55P82 and $\mathrm{P} 45 \mathrm{P} 55 . \mathrm{P} 55$ is the point at the left corner of mouth contour, and P82 is the point of the left face contour close to left eyecontour. If the facial expression is happy, AU12 is 
activated when the lip corner pulled up results in decreasing distance between lip corner and face contour on the same side on the face and these events activates AU12. The distance properties for each AU are observed for each level of facial expression's intensity of all facial data. The distance properties and the expected output which is the AU labelled as 0 or 1 . The distance difference and AU presence value $(0$ or 1) are the input for the SVM and NN classifier.

\section{EXPERIMENTS AND ANALYSIS RESULTS}

\section{Table 6.Average Correct Classification using SVM in} $\%$

\begin{tabular}{|c|l|c|c|c|}
\hline Expression & \multicolumn{1}{c}{ AU } & \multicolumn{1}{c}{ Linear } & \multicolumn{1}{c}{ Gaussian } & \multicolumn{1}{c|}{ Quadratic } \\
\hline \multirow{3}{*}{ Happy } & AU6 & 96.89 & 93.78 & 95.11 \\
\cline { 2 - 5 } & AU12 & 81.78 & 88.00 & 90.22 \\
\cline { 2 - 5 } & AU15 & 86.67 & 83.11 & 86.67 \\
\hline \multirow{4}{*}{ Sad } & AU1 & 98.67 & 98.22 & 100 \\
\cline { 2 - 5 } & AU4 & 85.78 & 94.67 & 94.22 \\
\cline { 2 - 5 } & AU17 & 88.44 & 87.56 & 92.00 \\
\cline { 2 - 5 } & AU25 & 94.67 & 92.00 & 93.77 \\
\hline
\end{tabular}

The BU-3DFE [21] data consists of four intensity levels for each frame. To obtain the difference of position for each facial point, the distance is calculated between the second, third and fourth intensity level frame to the first intensity level frame.

The experiments are carried out using 5-fold. Table 6 presents the result of the experiments. Average correct classification for all AUs is $99.91 \%, 99.91 \%$ and $99.89 \%$ is obtained using Linear, Gaussian and Quadratic kernel correspondingly. For AU1, $100 \%$ is achieved using the quadratic kernel while Linear and Gaussian achieved 99.99\% and $99.98 \%$ correspondingly. Each AU is correctly classified by the rate of more than $90 \%$ for the three kernels. Classification rate achieved using the quadratic kernel is 100\% (AU1), 90.22\% (AU12), 86.67\% (AU15) and 92\% (AU17). While for AU6, AU15 and AU25, the best kernel to use is linear with $96.89 \%, 86.67 \%$ and $94.67 \%$ respectively. Lastly, AU4 is greatly classified using a Gaussian kernel with $94.67 \%$, which is $0.45 \%$ greater than the Quadratic kernel.

AU1 (raised inner eyebrow) scores high classification rate for all experiments. The presence of AU1 is measured by increasing distance between the right corner point of left eye contour (P9) and right-most point of upper left eyebrow contour (P27). The distance between two points between male and female facial data has subtle differences. Therefore, all experiments obtained good results.

The distance from the left corner of mouth contour (P55) and left face contour (P82) of male and female have a significant difference. Thus, the decreased value in male data is less compared to female data. When testing is done using female data, many outliers are detected that eventually reduced the rate of classification.

However, AU12 (mouth corner pulled up) scores lower results across the AUs. AU12 is measured by the distance between P55P82 and P45P55. AU12 is activated if there is decreasing value in both distances. The distance from the left corner of mouth contour (P55) and left face contour (P82) of male and female have a significant difference. Thus, the decreased value in male data is less compared to female data. When testing is done using female data, many outliers are detected that eventually reduced the rate of classification. Both AU12 and AU15 have lower scores compared with the rest of the AUs. This is because distances for the facial points are only significant when the facial expression is high.

Table 7 presents the average classification results using $\mathrm{NN}$ and the hidden nodes are set to 10. Cross-Entropy (CE) presents the network performance which gives the inaccurate classification. The lower the value of $\mathrm{CE}$, the number of errors is reduced. Per cent Error (E) specifies the fraction of samples that are classified incorrectly. Zero values indicate no incorrect classification. The neuron is considered good at computing when the $\mathrm{CE}$ is a value is smaller or near to 0 .

AU25 scored the lowest CE for training (0.38\%), validation $(1.11 \%)$ and testing $(1.21 \%)$, as the facial motion of lips parted is significant. AU4 and AU6 scored high CE that are more than $3 \%$ for training, validation and testing except for AU6 validation scored is $2.71 \%$. AU4 activates lower eyebrow and the displacement of eyebrow facial points are sometimes unnoticeable. This is because the presence of AU4 is only obvious when the sad expression is at high intensity level. The other AUs scored lower CE, which is below $2 \%$. However, the CE percentage increased for all AUs during validation and testing. This is because the data size used is small which restrict the network to improve generalization.

Table 7.Average Correct Classification using NN

\begin{tabular}{|c|c|c|c|c|c|c|c|}
\hline \multirow{2}{*}{ Expression } & \multirow{2}{*}{ AU } & \multicolumn{2}{|c|}{ Training } & \multicolumn{2}{|c|}{ Validation } & \multicolumn{2}{c|}{ Testing } \\
\cline { 3 - 8 } & & $\mathbf{C E}$ & $\mathbf{E}$ & $\mathbf{C E}$ & $\mathbf{E}$ & $\mathbf{C E}$ & $\mathbf{E}$ \\
\hline \multirow{3}{*}{ Happy } & AU6 & 3.01 & 5.16 & 2.71 & 0 & 4.95 & 0 \\
\cline { 2 - 8 } & AU12 & 2.03 & 2.58 & 5.16 & 0 & 4.85 & 0 \\
\cline { 2 - 8 } & AU15 & 1.51 & 2.58 & 3.37 & 0 & 3.1 & 0 \\
\hline \multirow{4}{*}{ Sad } & AU1 & 1.66 & 0 & 5.16 & 0 & 4.71 & 0 \\
\cline { 2 - 8 } & AU4 & 3.37 & 0 & 9.44 & 0 & 9.3 & 0 \\
\cline { 2 - 8 } & AU17 & 2.15 & 0 & 4.76 & 0 & 4.78 & 0 \\
\cline { 2 - 8 } & AU25 & 0.38 & 0 & 1.11 & 0 & 1.21 & 0 \\
\hline
\end{tabular}

\section{VI.CONCLUSION}

In this work, $\mathrm{NN}$ and different kernels of SVM are used to classify the recognition rate of AU1, AU4, AU6, AU12, AU15, AU17 and AU25. As mentioned in the previous section, AU6, AU12 and AU15 are AUs present when a happy expression occurred. The rests of AUs are recognized in a sad expression. The performance of SVM is assessed using Linear, Gaussian and Quadratic kernel. By using any SVM kernels, it is consistent that AUs that are corresponded to sad expression has a high recognition compared to happy expression. This is may be due to thenumber of distance properties used for sad expression compared to happy expression. However, using NN, happy expression has a lower $\mathrm{CE}$ value on average compared to sad expression. It means that the number of errors occurred for happy expression is lower compared to the sad expression. 
In our future work, the number of features for each AU will be increased to achieve a more accurate result. The distance feature will be measure with a different set of facial points than the present technique used. For example, to detect AU12 (lip corner puller), more distance features could be added such as the distance between the tip of the chin and left corner of mouth contour.

\section{ACKNOWLEDGMENT}

This research is fully supported by Malaysian Ministry of Higher Education (MOHE) through Race Acculturation Collaborative Efforts RACE/1331/2016(4). The authors fully acknowledged MOHE and Universiti Malaysia Sarawak for the approved fund which makes this important research viable and effective.

\section{REFERENCES}

1. Y. Tian, T. Kanade and J.F. Cohn, "Facial Expression Analysis" in Handbook of Face Recognition, pp. 247-275, 2005, USA: Springer.

2. T. Fang, X. Zhao, O. Ocegueda, S.K. Shah and I.A. Kakadiaris, "3D Facial Expression Recognition: A Perspective on Promises and Challenges", Face and Gesture 2011, Santa Barbara, CA, pp. 603-610, 2011.

3. G. Sandbach, S. Zafeiriou, M. Pantic and D. Rueckert, "Recognition of 3D facial expression dynamics". Image and Vision Computing, 30(10), pp. 762-773, 2012

4. Y. Sun, M. Reale and L. Yin, "Recognizing Partial Facial Action Units Based On 3D Dynamic Range Data for Facial Expression Recognition", The 8th IEEE International Conference on Automatic Face \& Gesture Recognition, pp. 1-8, 2008.

5. N. Bayramoglu, G. Zhao and M. Pietikainen, "CS-3DLBP and Geometry Based Person Independent 3D Facial Action Unit Detection", In the Proceedings of the International Conference on Biometrics (ICB), pp. 1-6, 2013

6. P. Ekman and W.V. Friesen, "Measuring Facial Movement" in Environmental Psychology and Nonverbal Behavior, 1(1), pp. 56-75, 1976.

7. P. Lucey, J.F. Cohn, T. Kanade, J. Saragih, Z. Ambadar and I. Matthews, "The Extended Cohn-Kanade Dataset (CK+): A Complete Dataset for Action Unit and Emotion-Specified Expression", IEEE Computer Society Conference on Computer Vision and Pattern Recognition-Workshops, pp. 94 - 101, 2010.

8. K. Karthick, and J. Jasmine, "Survey of Advanced Facial Feature Tracking and Facial Expression Recognition", International Journal of Advanced Research in Computer and Communication Engineering, 2(10), 2013.

9. M. Pantic and L. Rothkrantz, “An Expert System for Recognition of Facial Actions and Their Intensity”, American Association for Artificial Intelligence, 2000

10. M. Valstar and M. Pantic, "Fully Automatic Facial Action Unit Detection and Temporal Analysis", Proceedings of the Conference on Computer Vision and Pattern Recognition Workshop, 2006.

11. H. Soyel and H. Demirel, "Facial Expression Recognition Using 3D Facial Feature Distances" in: Kamel M., Campilho A. (eds) Image Analysis and Recognition (ICIAR 2007), Lecture Notes in Computer Science, vol 4633. Springer, Berlin, Heidelberg, 2007.

12. H. Ujir, "3D Facial Expression Classification Using a Statistical Model of Surface Normals and a Modular Approach", University of Birmingham, Retrieved from eTheses Repository, 2013.

13. H. Ujir, C. Assan, I. Hipiny and D.N.F. Iskandar, "3D Measures and Geometric Facial Features Analysis on 3D Facial Action Unit Recognition, Advanced Science Letters, Vol. 21, No. 12, pp. 3703-3707(5), 2015.

14. H. Ujir and M. Spann, "Surface Normals with Modular Approach and Weighted Voting Scheme in 3D Facial Expression Classification", International Journal of Computer and Information Technology.Vol. 03 - Issue 05, pp. 909-918, 2014.

15. A.C.C. Ying, H. Ujir and I Hipiny, "3D facial expression intensity measurement analysis", in Zulikha, J. \& N. H. Zakaria (Eds.), Proceedings of the 6th International Conference of Computing \& Informatics, pp 43-48, 2017.
16. I. Hipiny and H. Ujir, "Measuring Task Performance using Gaze Regions", 9th International Conference on IT in Asia (CITA), pp.1-8, 2017.

17. I. Hipiny, H. Ujir, J.L. Minoi, S.F. Samson Juan, M.A Khairuddin and Sunar, S., "Unsupervised Segmentation of Action Segments in Egocentric Videos using Gaze", IEEE International Conference on Signal and Image Processing Applications (IEEE ICSIPA 2017), pp. 530-534, 2017.

18. J. M. Carroll and J. Russell, "Facial Expression in Hollywood's Portrayal of Emotion", Journal of Personality and Social Psychology. 72:164-176, 1997.

19. M. Beszédeš and P.F. Culverhouse, "Facial Emotions and Emotion Intensity Levels Classification and Classification Evaluation", British Machine Vision Conference, 2007.

20. K.K Lee and Y. Xu, "Real-Time Estimation of Facial Expression Intensity", IEEE International Conference on Robotics and Automation, pp. 2567-2572, 2003.

21. L. Y in, X. Wei, Y. Sun, J. Wang and M. J. Rosato, “A 3D Facial Expression Database for Facial Behavior Research", 7th International Conference on Automatic Face and Gesture Recognition, pp. $211-216,2006$.

22. Y. Tian, T. Kanade and J.F. Cohn, "Recognizing Action Units for Facial Expression Analysis", IEEE Transactions on Pattern Analysis and Machine Intelligence, 23(2), pp. 97 - 115, 2001.

23. P. Michel, and R. E. Kaliouby, "Facial Expression Recognition using Support Vector Machines", In Proceedings of the International Conference on Human-Computer Interaction (HCI): Human-Computer Interaction, Theory and Practice, (2), pp. $93-$ 94, 2003.

24. A. Kapoor, Y. Qi and R.W. Picard, "Fully Automatic Upper Facial Action Recognition", IEEE International Workshop on Analysis and Modelling of Faces and Gestures, pp. 195, 2003.

25. H. Li, J.M. Morvan and L. Chen, "3D Facial Expression Recognition Based on Histograms of Surface Differential Quantities", In the Proceedings of the 13th International Conference on Advanced Concepts for Intelligent Vision Systems (ACIVS'11), pp. 483 - 494, 2011

26. Manisha, J. Singh and N.R. Prakash, "Facial Expression Recognition Using Neural Network", International Journal of Computer Science and Information Technologies (IJCSIT), Vol. 6 (3), pp. 3249-3251, 2015.

27. J.R. Mangrolia and J.B. Patel, "Automatic Facial Expression Recognition using Support Vector Machine and Neural Network", International Journal Computer Technology \& Application, (5), pp. 587 - 593, 2014.

28. S. Berretti, N. Werghi, A.D. Bimbo and P. Pala, "Matching 3D Face Scans using Interest Points and Local Histogram Descriptors". Computer \& Graphics, 37(5), pp. 509 - 525, 2013.

29. M. Daoudi, A. Srivastava and R.Veltkamp, "3D Face Modeling, Analysis and Recognition", 1st Ed. Singapore: John Wiley \& Sons, 2013.

30. A. Savran and B. Sankur, "Automatic Detection of Facial Actions from 3D Data", IEEE 12th International Conference on Computer Vision Workshops, pp. 1993 - 2000, 2009.

31. R. Sprengelmeyer and I. Jentzsch, "Event Related Potentials and the Perception of Intensity in Facial Expressions", Journal of Neuropsychologia, Vol. 44, pp. 2899-2906, 2006.

32. R. J. Vidmar. (1992, August). On the use of atmospheric plasmas as electromagnetic reflectors. IEEE Trans. Plasma Sci. [Online]. 21(3). pp. 876-880. Available: http://www.halcyon.com/pub/journals/21ps03-vidmar

\section{AUTHORS PROFILE}

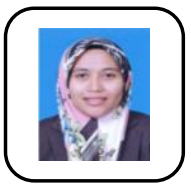

Norhaida Hussain works as a lecturer at Malaysia Polytechnic. She is currently a $\mathrm{PhD}$ student at Faculty of Computer Science and Information Technology, Universiti Malaysia Sarawak. She received her BSc in Computer Science from Universiti Sains Malaysia and her master's degree in Advanced Information Technology from Universiti Malaysia Sarawak. Her research interests include crowd simulation, crowd surveillance and crowd steering. Her current research focuses on path planning and crowd simulation in evacuation environments. 
Dr Hamimah Ujir is currently a Senior Lecturer at the Faculty of Computer Science and Information Technology (FCSIT), Universiti Malaysia Sarawak (UNIMAS). She received her $\mathrm{PhD}$ from University of Birmingham, United Kingdom. Hamimah's research interests lie in the interdisciplinary field of computer vision, with related interests being in computer graphics, image processing, and mathematical methods. Her previous and current works include computer visions, physical simulation, facial analysis and academic quality. Currently Hamimah is the Deputy Director for Accreditation and Quality Management department at Centre of Quality Assurance and Academic Development, UNIMAS.

Dr Irwandi Hipiny is a Senior Lecturer at Universiti Malaysia Sarawak and currently serving as the Programme Coordinator for WC09 Multimedia Computing programme. He holds a BSc (2003) as well as an MSc (2007) in Computer Science from Universiti Teknologi Malaysia, and a PhD (2014) in Computer Vision from University of Bristol. Prior to his current appointment, Irwandi was a recipient of the Ekpress UTM-MARA scholarship (1999 - 2003) and was a MOSTI's National Science fellow (2003 - 2007). Irwandi's research interests are computer vision, pattern recognition and educational games.

Dr Jacey-Lynn Minoi is a Senior Lecturer in Computer Science and a Research Fellow at the Institute of Social Informatics and Technological Innovation at the Universiti Malaysia Sarawak. She was previously a Research Associate at the Royal College of Surgeon under the Wellcome Trust, and a Visiting Professor at Glasgow University, UK. Her research interests are in data analytics and image processing. 